\title{
21
}

\section{A simulation study of a wireless bandwidth reservation multiple access protocol for multimedia traffic}

\author{
Zhang, Z., Habib, I. and Saadawi, T. \\ E.E. Department, City University of New York \\ New York, NY 10031, Tel. (212) 650-8378, fax (212) 650-8249 \\ e-mail zzhang@ee-mail.engr.ccny.cuny.edu
}

\begin{abstract}
Bandwidth reservation multiple access protocol (BRMA) resolves access contentions for mobile users in wireless local networks. Bandwidth is assigned to each user dynamically at frame level. In each frame, request channels are deterministically assigned to each user and are therefore contention-free. Data channels are assigned dynamically by base station and are therefore collisionfree. In this paper, BRMA is studied for multimedia traffic through simulations. Traffic patterns of different kinds, such as voice, data, real time high activity video (MPEG movie Star Wars) are used to study the protocol performance. With a proper admission control and with proper design of protocol parameters (service ratio, frame length, etc), traffic with different quality of service (QoS) requirements can be supported.
\end{abstract}

\section{Keywords}

Wireless networks, multiple access protocol, BRMA, multimedia

\section{INTRODUCTION}

To provide the wireless users with access to the advanced information services, a wireless system should be able to accommodate multimedia traffic. In a wireless system, the system structure and performance largely depend on the multiple access control (MAC) protocol that is used. So far it is still a common difficulty for a wireless system to provide the multimedia variable bit rate (VBR) traffic 
with reliable service and yet maintain an efficient channel utilization. TDMA, as a mature technology in the wired systems for many years, is adapting itself to compete against the rather new CDMA to be the dominant wireless multiple access control technology in the future. However, most TDMA versions, including the multiservice dynamic reservation TDMA scheme studied in Wilson et al.,(1993), do not utilized the wireless bandwidth as efficiently as the wide band CDMA especially for bursty traffic. One major reason of this is that the effect of the multiplexing gain is not maximally taken advantage of. For example, the voice activity detectors are generally not used in most TDMA systems due to the difficulty of the implementation.

Goodman et al proposed a Slotted-Aloha-reservation type packet reservation multiple access (PRMA) scheme to enhance the performance of TDMA for periodic traffic pattern such as in the case of voice conversation, see Goodman et al., (1989). After its proposal PRMA attracted much attention and was studied by many other researchers, see Goodman and Wei (1991), Nanda, (1990) and Wu et al., (1994). PRMA utilizes the speech detectors and allows a wireless user to reserve bandwidth only during talkspurts rather than during the entire period of conversation. This approach does improve the performance significantly for voice traffic. However, due to the collisions during the Slotted Aloha process before each reservation, PRMA does not perform efficiently for other types of traffic which are not periodic or which have very short bursts.

Distributed wireless random access control protocols, such as Aloha, Slotted Aloha, PRMA and its variants, work efficiently for systems with low speeds, long propagation delays and light traffic loads. They are simple to implement and provide low packet delays under light load traffic. However they cannot achieve high channel utilizations and have stability problems under heavy load. For systems with high speeds, short propagation delays and heavy loads, the centrally controlled wireless access protocols are usually better solutions at the cost of high processing.

An ATM-friendly bandwidth reservation multiple access protocol (BRMA) was proposed for wireless local high speed systems with short propagation delays, see Zhang, et al., (1996). Designed to support variable bit rate (VBR) traffic as well as other multimedia traffic, BRMA was studied through both queueing analysis and simulations for voice and data traffic in Zhang, et al., (1996). The result was that it performed better than PRMA and classical TDMA in the high speed heavy load systems.

In this paper we further study the BRMA for multimedia traffic. In section 2, we present a overview of wireless systems and the basic BRMA protocol. The applications of BRMA for two or more classes of traffic is discussed in section 3. In section 4 , we study BRMA for voice and data traffic to further compare with the PRMA. In section 5, we simulate the protocol performance for voice and high activity video. Then the conclusion of this paper is given in section 6 . 


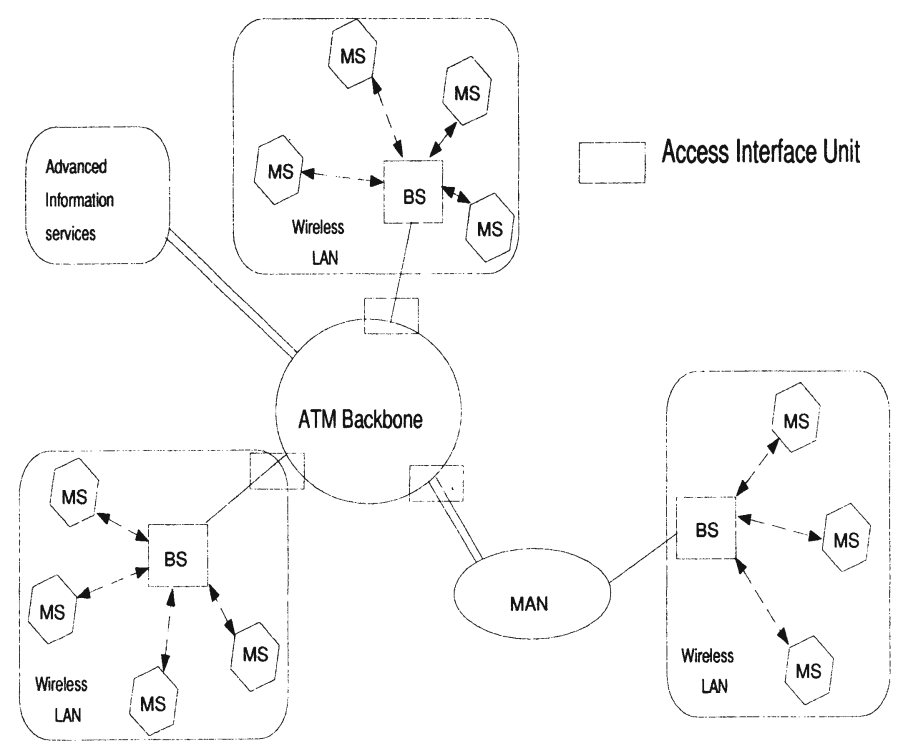

Figure 1 A system overview.

\section{A WIRELESS SYSTEM OVERVIEW AND BASIC BRMA}

As shown in Figure 1, wireless systems are usually connected to a wired backbone network through base stations (BS). In each wireless LAN, a number of mobile stations (MS) share the common uplink wireless channel. The downlink channel, the one from BS to MS, is an efficient broadcast channel and therefore not discussed here.

With BRMA, the uplink channel time is divided into frames of equal length. There are $\mathrm{K}$ data slots (data channel) and $\mathrm{N}$ mini slots (request channel) in each frame, where $\mathrm{N}$ equals to the number of MS that are connected to the BS, and where $\mathrm{K}$ is a design parameter. See Figure 2 for the frame structure. In each frame, each mini slot is deterministically assigned to one MS that has established a connection in the network. In each frame, each MS uses its preassigned mini slot to send its request for data slots in the current frame basing on the number of packets that are waiting in its buffer. At the end of the mini slots the BS knows the total number of requested data slots in this frame and broadcasts the data slots assignment immediately. Then each MS transmits its data packets in its assigned data slots. Apparently for this protocol to work efficiently, the packet propagation delay has to be small, such as in the case of micro cell or pico cell. 


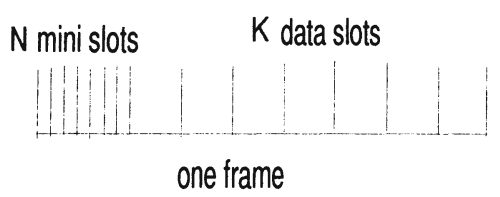

Figure 2 A BRMA frame structure.

As a central controller inside the wireless LAN, the BS keeps a connection table for all MS. When a MS is connected to or disconnected from the wireless LAN, the BS updates this information as well as the mini slots assignment instantly. In the BS, a standard ATM header will replace the short herder of each data packet from the MS to the backbone network. Therefore all outgoing packets are ATM cells.

The data slot assignments are made based on the availability and request of the data slots in each frame. If the total request for data slot in this frame does not exceed $\mathrm{K}$, then every MS will be assigned as many data slots as it requests. However if the total requested number exceeds $\mathrm{K}$, then some MS may not get as many data slots as they need in this frame. If one MS is assigned less data slots than it requested, some of its packets will have to wait to be transmitted in the following frames. If the data packets are delay sensitive, the packet loss may occur.

To guarantee the QoS and limit the packet loss and delay, a minimum number data slots per frame is guaranteed to the MS upon request. Such minimum number is predetermined for each MS during call setup based on its traffic and QoS requirement. With such provision of minimum bandwidth, a MS will always get at least this amount of bandwidth upon request even in time of congestions. To avoid the wireless LAN being overloaded, an admission control scheme such as the one described in Habib and Saadawi (1992) should be applied.

Above data slots assignment procedure is repeated every frame and the bandwidth assignments are dynamic at the frame level. Apparently BRMA takes a good advantage of the multiplexing gain at the cost of the mini slots overhead. It suits not only the periodic traffic but also other types of multimedia traffic.

\section{BRMA SUPPORTING TWO CLASSES OF TRAFFIC}

Now we consider two groups (A and B) of wireless users that are connected to the BS. The number of wireless users in group $A$ and group $B$ are $\mathrm{N}_{\mathrm{A}}$ and $N_{B}$ respectively. Each group requires a different class of QoS. There are many definitions for QoS. Here we consider only the packet loss rate and the average packet delay. The packet loss occurs when a packet is delayed more than a limit or equivalently when the buffer overflows. For example, for voice conversations, QoS is usually met when packet loss rate is under $1 \%$ for the delay limit of $32 \mathrm{~ms}$. 
To guarantee the QoS to the two groups of wireless users, in each frame $\mathrm{K}_{\mathrm{A}}$ and $\mathrm{K}_{\mathrm{B}}$ data slots are guaranteed to group $\mathrm{A}$ and $\mathrm{B}$ respectively. Apparently we have:

$$
\begin{aligned}
& N=N_{A}+N_{B} . \\
& K=K_{A}+K_{B} .
\end{aligned}
$$

This data slots partitioning (or called service ratio $\mathrm{K}_{\mathrm{A}}: \mathrm{K}_{\mathrm{B}}$ ) is determined when connections in group A and B are established, based on the traffic conditions and required QoS. When traffic conditions change, for example, when new calls are admitted or when existing calls terminate, the values of $\mathrm{K}_{\mathrm{A}}$ and $\mathrm{K}_{\mathrm{B}}$ need to be changed accordingly.

Due to the frame level dynamic assignment, in any particular frame, however, the data slots used by group $A$ and $B$ may not be exactly $\mathrm{K}_{\mathrm{A}}$ and $\mathrm{K}_{\mathrm{B}}$ respectively. When group $A$ cannot use up the $K_{A}$ data slots and when group $B$ needs more than $\mathrm{K}_{B}$ data slots, group $B$ may utilize the unused portion of the $\mathrm{K}_{A}$ data slots that are guaranteed to group $\mathrm{A}$. This is true vice versa. One example of this is shown in Figure 3. Figure 4 shows another example when the service ratio $K_{A}: K_{B}$ changes.

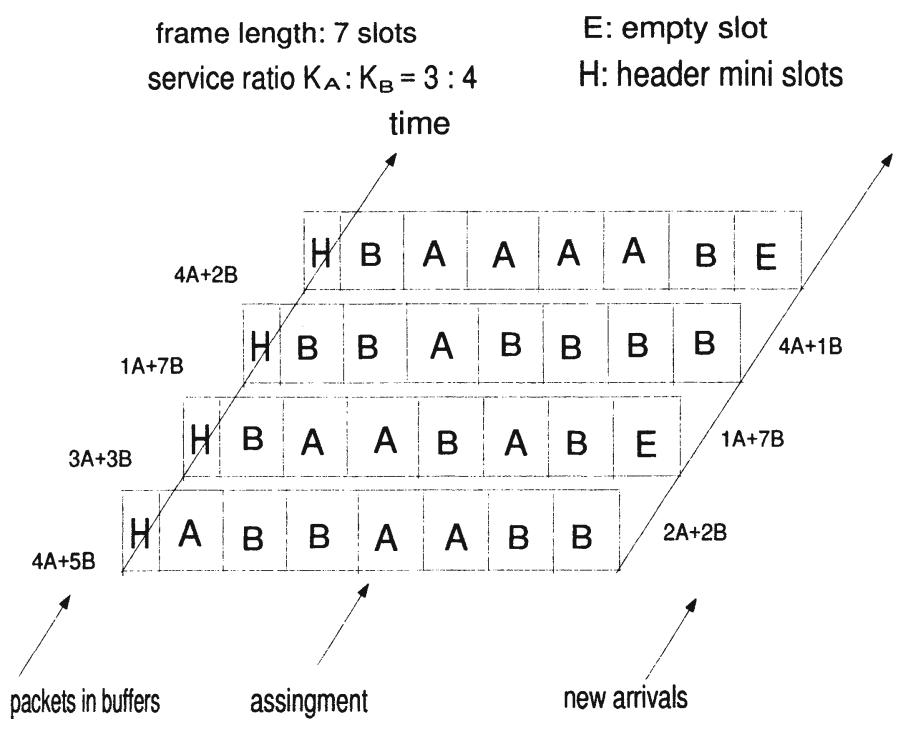

Figure 3 An example of data slots assignment. 


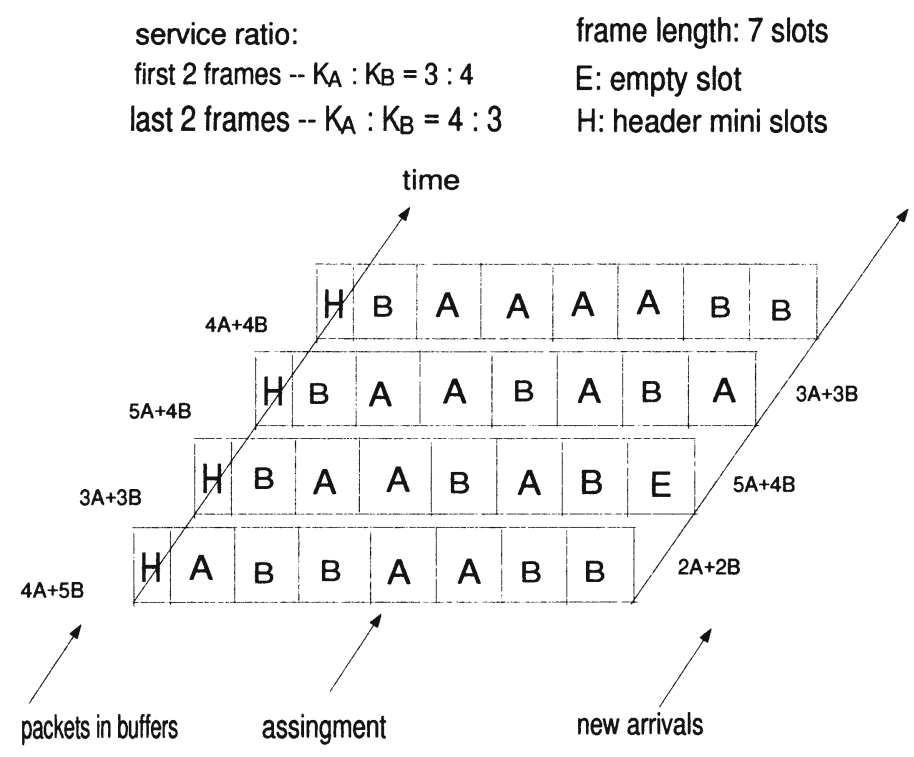

Figure 4 Another example of data slots assignment.

In the frame structure, the value of $\mathrm{K}$ is another design parameter. When $\mathrm{K}$ is too small, a large portion of the frame will be used by overhead (mini slots) and therefore the protocol efficiency is low. When $\mathrm{K}$ is too large, packets that arrive early in a frame will be delayed too long before data slots are requested for them therefore they will be excessively delayed. Thus an optimal value of $\mathrm{K}$ exists. The eptimal value of $\mathrm{K}$ usually depends on the traffic condition and the QoS requirements, among others.

As we have seen so far, the number of classes of traffic that BRMA can support is by no means limited to two. For simplicity, we only consider the case of two classes of traffic in this paper.

\section{BRMA SUPPORTING VOICE AND DATA}

In this section we will do additional comparative study on BRMA for voice and Poisson data traffic, to compare with PRMA. In Wu et al., (1994), PRMA performance was further studied for voice and data traffic. A Markovian analysis was also presented in their paper. In Wu et al., (1994), voice sources are modeled by the on-off sources, and data packets are generated randomly. The inter-arrival time (length of TH mode) of the data packets is exponentially distributed with mean of $0.32 \mathrm{~s}$. The data terminals generate only one single packet each time. The parameters of the system studied in Wu et al., (1994) are given in Table 1. The average delay for both voice and data packets were obtained when the packet transmission probabilities were optimized. 


\begin{tabular}{|l|l|}
\hline Variable & Nominal value \\
\hline mean talkspurt duration for voice (s) & 1.00 \\
\hline mean silent duration for voice (s) & 1.35 \\
\hline mean duration in TH mode for data (s) & 0.32 \\
\hline channel rate (kbps) & 360 \\
\hline speech coding rate for voice (kbps) & 32 \\
\hline packet size for voice and data (bits) & 576 \\
\hline slot duration (ms) & 1.6 \\
\hline frame duration (ms) & 16 \\
\hline Number of slots per frame & 10 \\
\hline priority: Pv/Pd & 15 \\
\hline number of voice terminals Mv & $5,10,15$ \\
\hline
\end{tabular}

Table 1 System and traffic parameters

We simulated BRMA delay performance for the same wireless system studied in Wu et al., (1994). We used the same traffic parameters as shown in Table 1, wherever applicable. In our study the service ratios are chosen as $K_{V}: K_{D}=K: 0$, which means that data packets may be transmitted only when all voice packets are transmitted and data slots in a frame are still available. Each mini slot is chosen as two bytes. Each data packet has a header of five bytes. Frame lengths are all optimized. The optimized frame length here is defined as the one that minimizes the average voice packet delay while guaranteeing that the packet loss rate for voice is under $1 \%$.

For the traffic load shown in Figure 5, the voice packet delay performance of PRMA and BRMA are provided in Figure 6. When voice traffic load is light $(\mathrm{Mv}=5,10)$ and when the number of data terminals is large, PRMA provides smaller average packet delay for voice. The reason of this is as following: when voice traffic load is light, packet collisions are less likely to happen at the beginning of the bursts. Once the reservation is made, following packets from the same terminal do not suffer from additional delay to the end of the burst. For BRMA, there are considerably large overhead especially due to the data terminals (each of which needs one mini slot). 


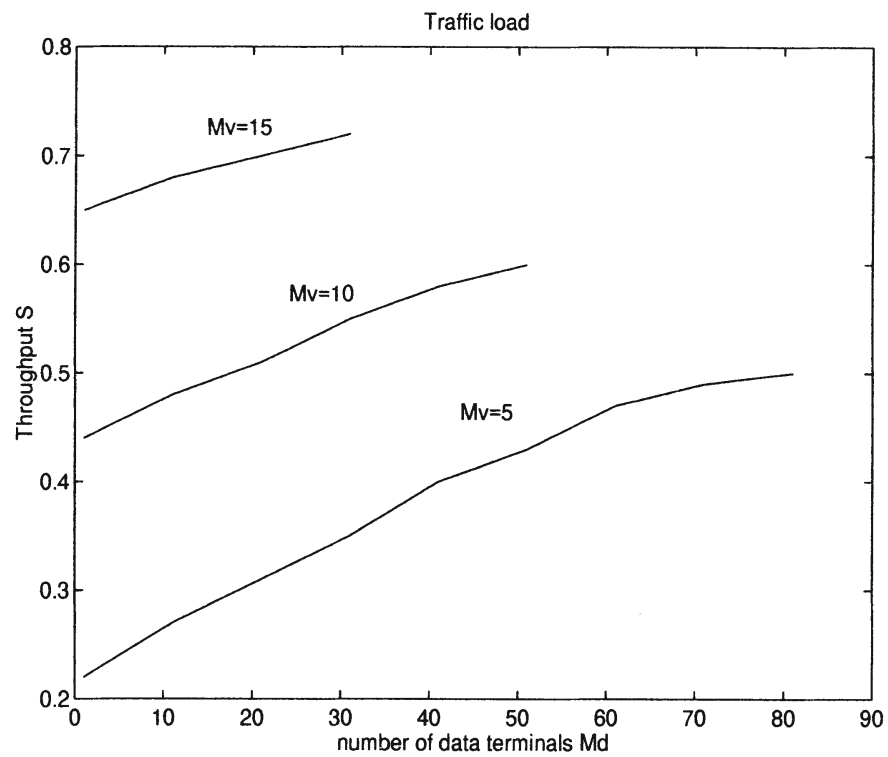

Figure 5 Traffic loads.

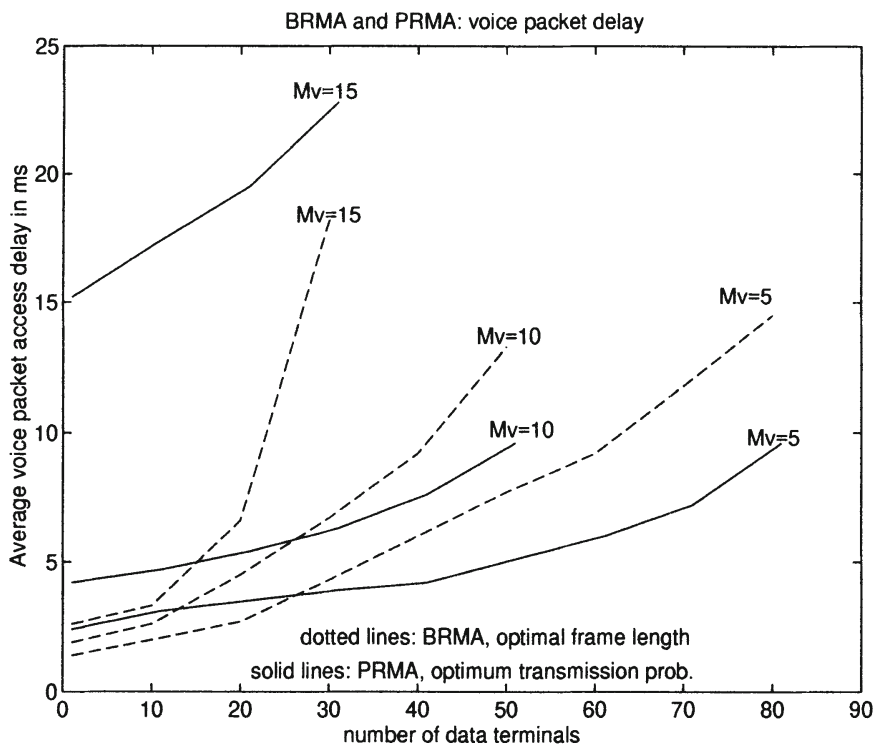

Figure 6 Voice packet delay performance for PRMA and BRMA. 


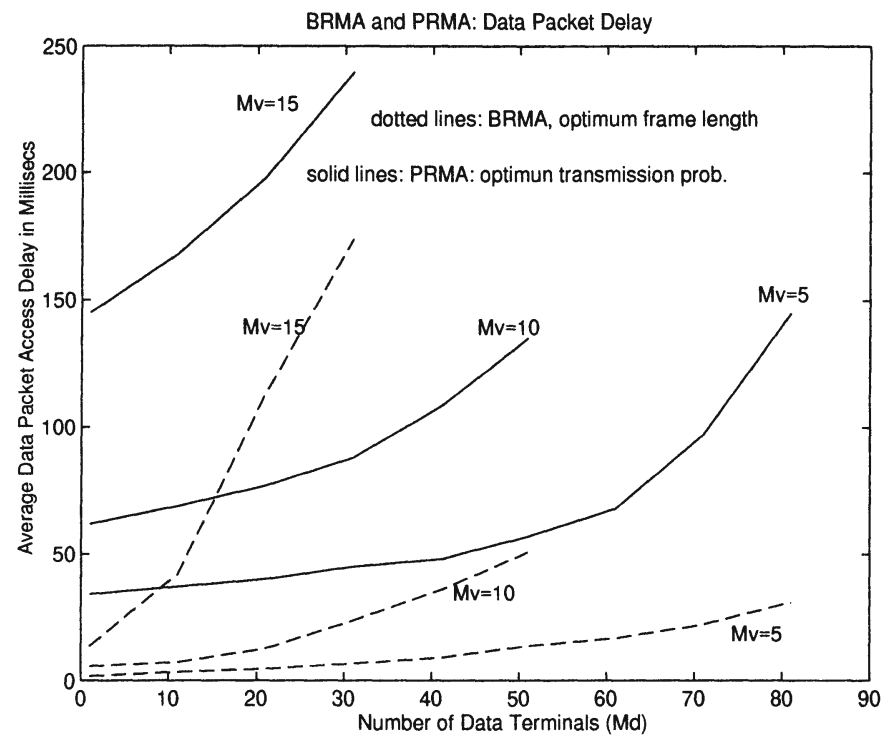

Figure 7 Data packet delay performance for PRMA and BRMA.

When the voice traffic load is higher ( $\mathrm{Mv}=15$ here), PRMA will produce higher delay than the BRMA for voice packets. This is because more voice packets collisions happen before a reservation can be made. From here we conclude that BRMA is more efficient when there are fewer wireless users in the system and each user is generating rather heavy traffic. In this case less overhead is necessary.

Figure 7 shows the data packet average delay for both PRMA and BRMA. It is clear that for $\mathrm{Mv}=5,10$, and $15, \mathrm{BRMA}$ provides a better performance than PRMA for data packets. This is because that in PRMA every single data packet that is transmitted has to go through the Slotted Aloha process and no reservation can be made.

This concludes our comparative study of PRMA and BRMA: Firstly, BRMA works better for heavy load systems and PRMA works better for light load systems. This is not surprising because BRMA is a centralized control protocol while PRMA is a distributed one. Secondly, BRMA performs consistently efficiently for both periodic traffic ( such as voice) and non-periodic traffic (such as data), while PRMA performs efficiently for the long-burst periodic traffic but not as efficiently for short-burst non-periodic traffic. 


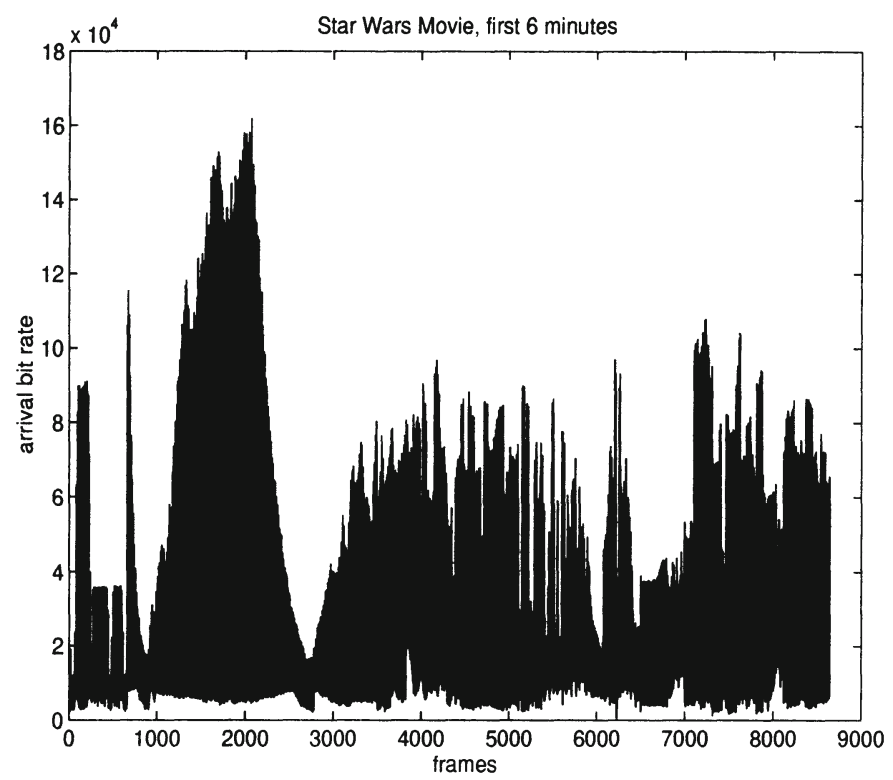

Figure 8 High activity video packet arrivals.

\section{BRMA SUPPORTING HIGH ACTIVITY VIDEO AND VOICE}

High activity video such as movies will be an important part of future multimedia traffic, therefore is worth investigating. It usually has high bit rate as well as high burtiness.

Currently many multimedia services in which stored media objects can be retrieved on demand by end users adopt the popular international encoding standard Motion Picture Experts Group (MPEG), see WG 11. In MPEG, video is coded into a sequence of Intracoded frame (I), Predictive frame (P) and Bidirectional frame (B). In this paper we study the BRMA performance for high activity video using the MPEG coded movie Star Wars as one example.

Our MPEG movie Star Wars is obtained from the public domain of Bellcore and the acknowledgment is due to Mark Garrett of Bellcore and Professor Martin Vetterli of the University of California at Berkeley. It is MPEG I with frame sequence of I B B P B B P B B P B B. 24 frames are generated in each second. See Garrett, (1993) for detail description of this set of data. In this study, video packets are generated uniformly in each frame interval of $1 / 24$ seconds.

In our study we take a part of the Star Wars movie to be a sample of high activity video source. Figure 8 shows the arrival bit rate (in bits/frame ) of this source. Some statistics of this sample is shown in Table 2. We shift the sample by some frames to obtain additional video sources so that these sources are very 
similar statistically. Figure 9 shows the index of dispersion for count (IDC) of the video. We see that the IDC of ten sources is much lower than that of one source, which implies that the traffic become "smoother" when multiplexed.

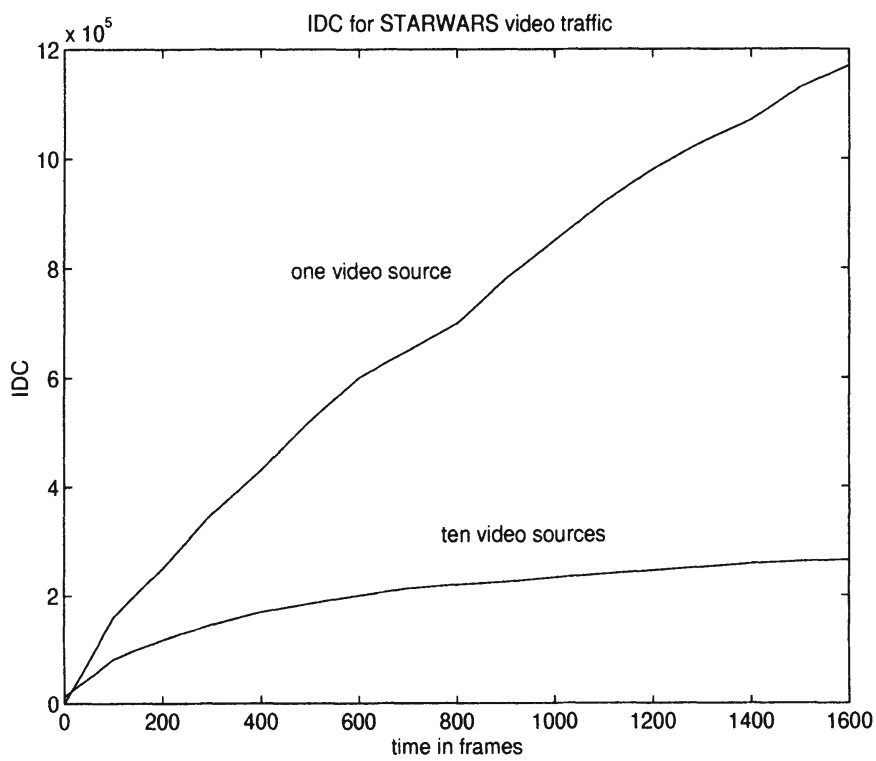

Figure 9 IDC for high activity video traffic.

\begin{tabular}{|l|l|l|l|}
\hline peak (bit/frame) & mean (bit/frame) & variance & peak/mean \\
\hline 161,726 & 16,679 & $3.6 \mathrm{e}+12$ & 9.7 \\
\hline
\end{tabular}

Table 2 Traffic statistics for high activity video 


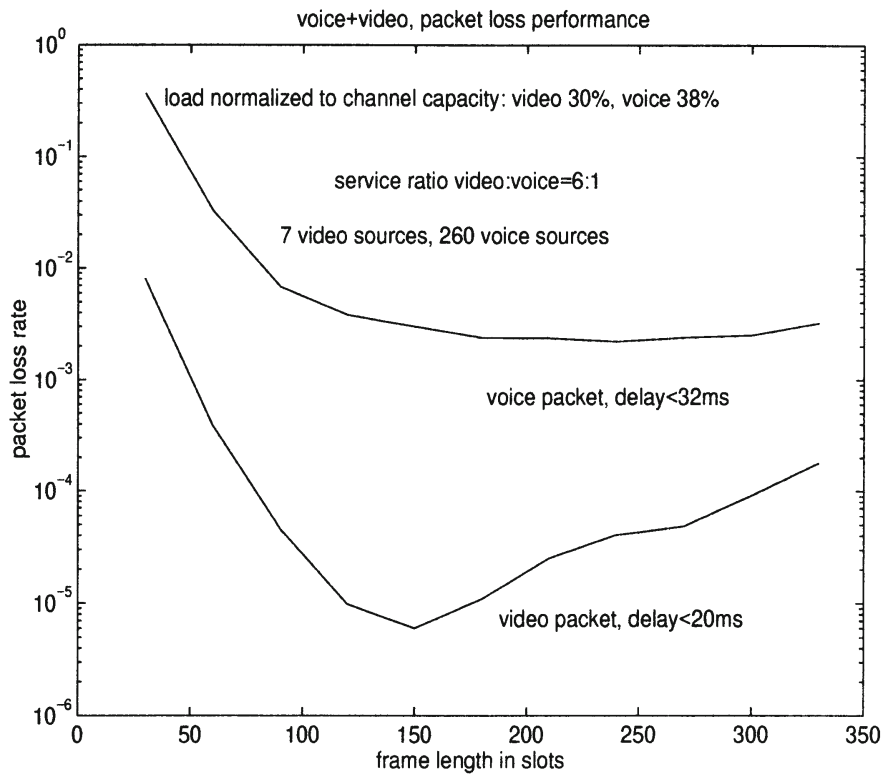

Figure 10 Packet loss performance for voice and video.

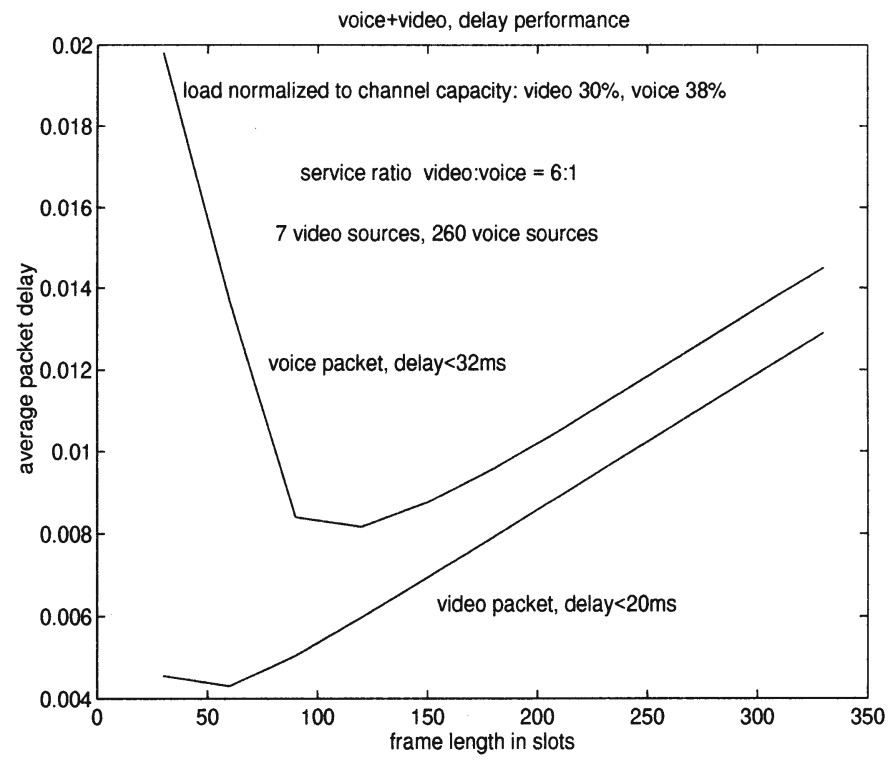

Figure 11 Packet delay performance for voice and video. 
Figure 10 and 11 show the packet loss and delay performance of BRMA for high activity video and voice at the channel capacity of $10 \mathrm{Mbps}$. When the frame length is around 150 slots, 7 video and 260 voice sources can be supported with QoS that the packet loss rate for video and voice are under 1.0e- 05 and 1.0e-02 respectively while the packet delay limits are $20 \mathrm{~ms}$ and $32 \mathrm{~ms}$ respectively. For traditional wireless TDMA, i.e., if bandwidth is dedicated to each user rather than shared, a $10 \mathrm{Mbps}$ system can only support

$$
N=\frac{10240000}{24 \times 161726} \approx 2.6
$$

such high activity video connections. From here we see that a significant multiplexing gain is indeed achieved.

Figure 11 shows that the delays for video and voice are small when frame length is near 150 slots. Therefor the optimal frame length is near 150 slots.

Now we study the impact of service ratio on the protocol. The service ratio here sets a boundary between the resources allocated to video and voice. This boundary is flexible, as bandwidth assignment depends also on the traffic conditions. As shown in Figure 12, for the connected 7 video and 310 voice sources, the system with channel capacity $10 \mathrm{Mbps}$ can guarantee the above QoS when the service ratio video : voice is between the values of 7.5 and 10 .

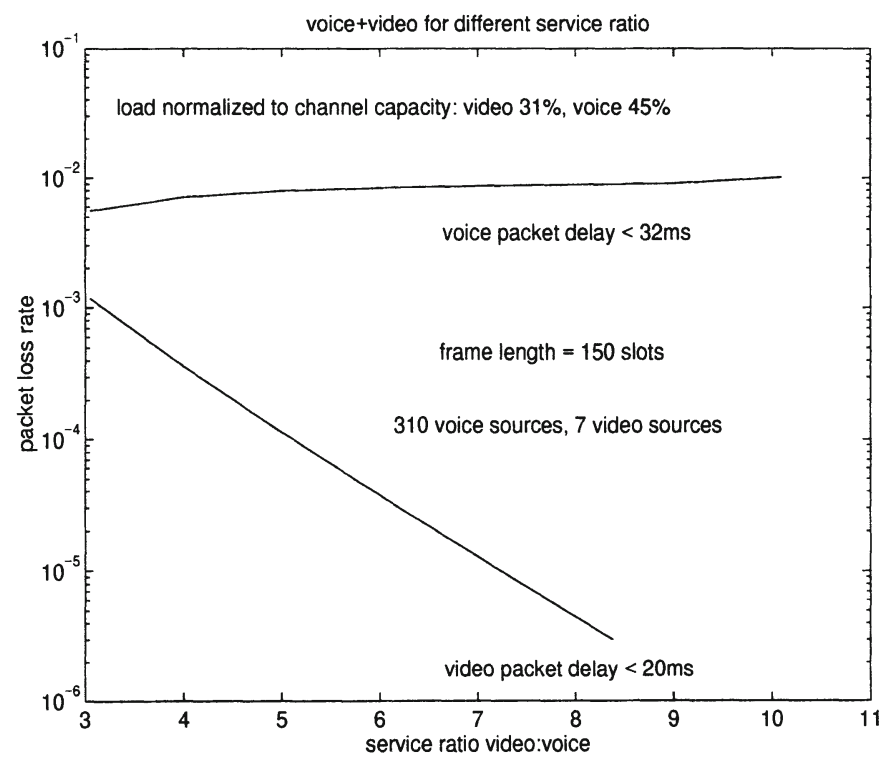

Figure 12 Packet loss performance for different service ratio. 


\section{CONCLUSIONS}

In this paper we studied the Bandwidth Reservation Multiple Access (BRMA) protocol performance for multimedia wireless systems. We demonstrated that the protocol can support multiple classes of traffic including data, voice, and high activity video ( such as MPEG movie of Star Wars). Proper frame length and service ratio guarantees that the protocol works efficiently. Admission control and minimum bandwidth that are reserved at call setup stage guarantee the QoS to each MS. It is also demonstrated in the paper that BRMA can achieve a high multiplexing gain for all types of traffic that are studied.

\section{REFERENCES}

Wilson, N., Ganesh, R., Joseph, K. and Raychaudhuri, D. (1993) "Packet CDMA versus Dynamic TDMA for multiple access in an integrated voice/data PCN," IEEE JSAC, 11, 870-884.

Garrett, M. (1993) "Contributions toward real-time services on packet networks," $\mathrm{Ph} . \mathrm{D}$ Dissertation, Columbia University.

Goodman, D., Valenzuela, R. Gayliard, K. and Ramamurthi, B. (1989) "Packet reservation multiple access for local wireless communications," IEEE Transaction on Communications, 37, 885-889.

Goodman, D. and Wei, S. (1991) "Efficiency of packet reservation multiple access," IEEE Transaction on Vehicular Technology, 40, 170-176.

Habib, I. Saadawi, T. (1992) "Dynamic bandwidth and access control of virtual paths in ATM networks," proceeding of IFIP 3rd International Conference on High Speed Networks, Belgian.

Nanda, S. (1990) "Analysis of packet reservation multiple access: voice and data integration for wireless networks," Proceedings of GLOBECOM'90, San Diego, CA, 1984-1988.

WG 11, "MPEG 1: coded representation of picture, audio and multimedia/hypermedia information," ISO/IEC JTC 1/SC 29, Nov. 1991

Wu, G., Mukumoto, K. and Fukuka, A. (1994) "Analysis of an integrated voice and data transmission system using packet reservation multiple access," IEEE Transaction on Vehicular Technology, 43.

Zhang, Z., Habib I. and Saadawi, T. (1996) "A bandwidth reservation multiple access protocol for wireless ATM local networks," MILCOM 96, October 1996, McLean, Virginia 


\section{BIOGRAPHY}

Zhang, Zhiguang received his M.E. and B.E. degrees from Huazhong University of Science and Technology in China in 1989 and 1984, respectively. Currently he is a Ph.D. candidate and a teaching fellow in City University of New York. His research focuses on wireless communications.

Habib, Ibrahim is an assistant professor in City University of New York. He received his B.S. degree from Ain Shams University in Egypt, 1981. In 1984 he received an M.S. degree from Polytechnic University of Brooklyn. In 1991 he received his Ph.D. degree from City University of New York. Dr. Habib's research interests include ATM networks and applications of neural networks in high speed networks.

Saadawi, Tarek is currently a professor in City University of New York. He received his B.S. and M.S. degrees from Cairo University in 1973 and 1975, respectively. In 1980 he received a Ph.D. degree from University of Maryland. Dr. Saadawi's research interests are high-speed and multimedia networks. 